\title{
edificio para viviendas, En Barcelona
}

\author{
F. BARBA CORSINI, Dr. arquitecto
}

J. CANOVES Y M. CASANELLAS, arquitectos colaboradores

\begin{abstract}
simopsis El edificio contiene: en el sótano, un guardacoches; en la planta baja, una zona de juegos común para los niños de todas las viviendas, además de los vestíbulos. El resto de la superficie edificada está destinado a viviendas, de unos $200 \mathrm{~m}^{2}$, construidos con distribución funcional y adecuada para familias medias ya constituidas.

$123-87$

Fue dedicada una especial atención a la composición de las fachadas, que ofrecen, entre otros atractivos, el de su elegante sencillez.
\end{abstract}

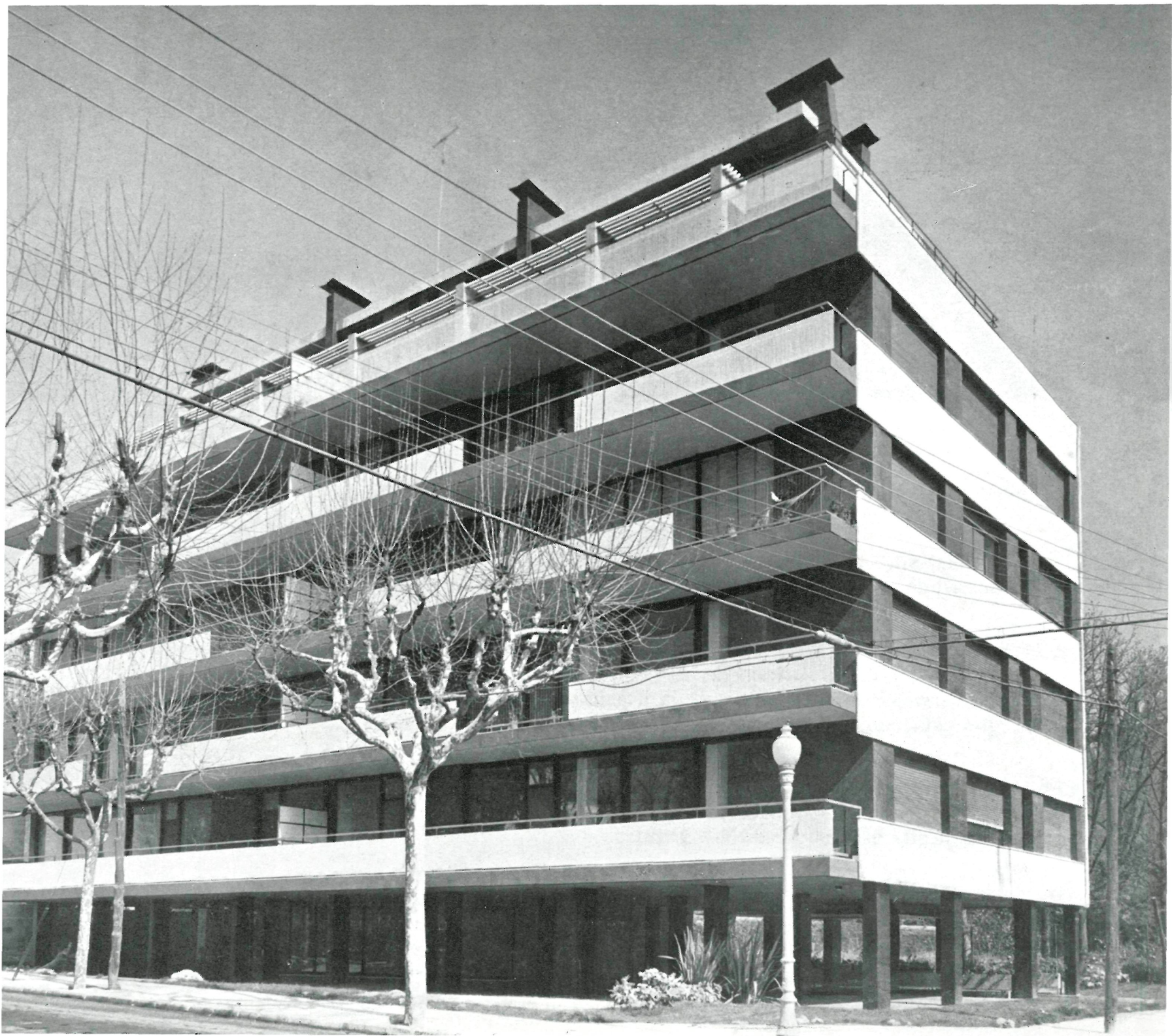




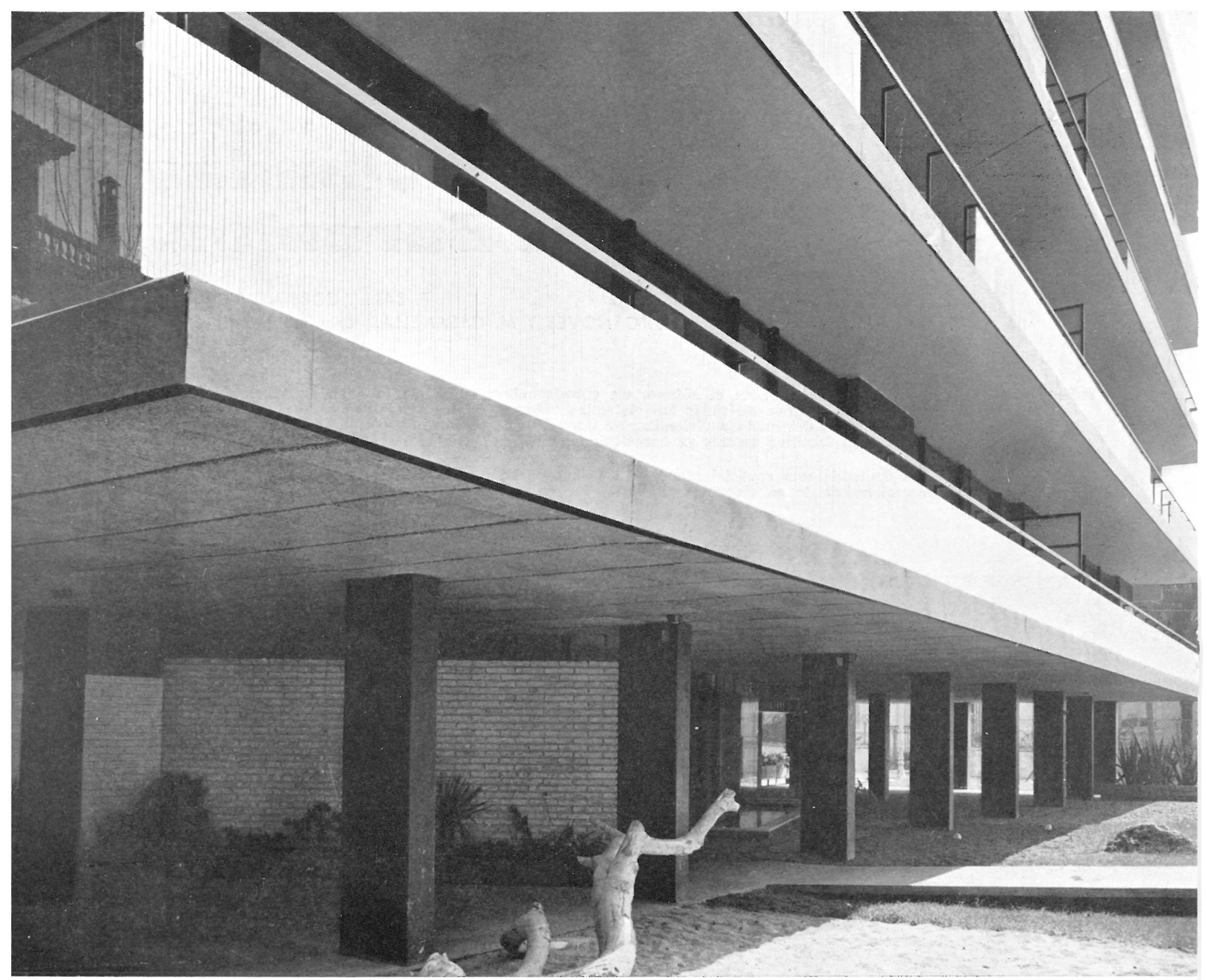

Sobre el solar sito en la calle Escuelas Pías, números 43 y 45 (Tres Torres), de Barcelona, ha sido construida esta casa para venta por pisos de primera categoría.

A la misma se ingresa a través de: una entrada al vestíbulo principal, desde el que se accede al ascensor, y de la entrada de servicio, por la parte posterior, que va a parar al vestíbulo de servicio, situado al pie de la escalera; ambas controladas por la cabina del conserje, que está comunicada con su vivienda.

El edificio contiene: en el sótano, un guardacoches; en planta baja, una zona de juegos común para los niños de todas las viviendas, además de los vestíbulos. El resto de la superficie edificada está dedicado a viviendas.

Ya en la fase de proyecto, la «forma» del terreno llevó automáticamente a los arquitectos a inclinarse por una solución de planta rectangular, con dos viviendas por rellano. 

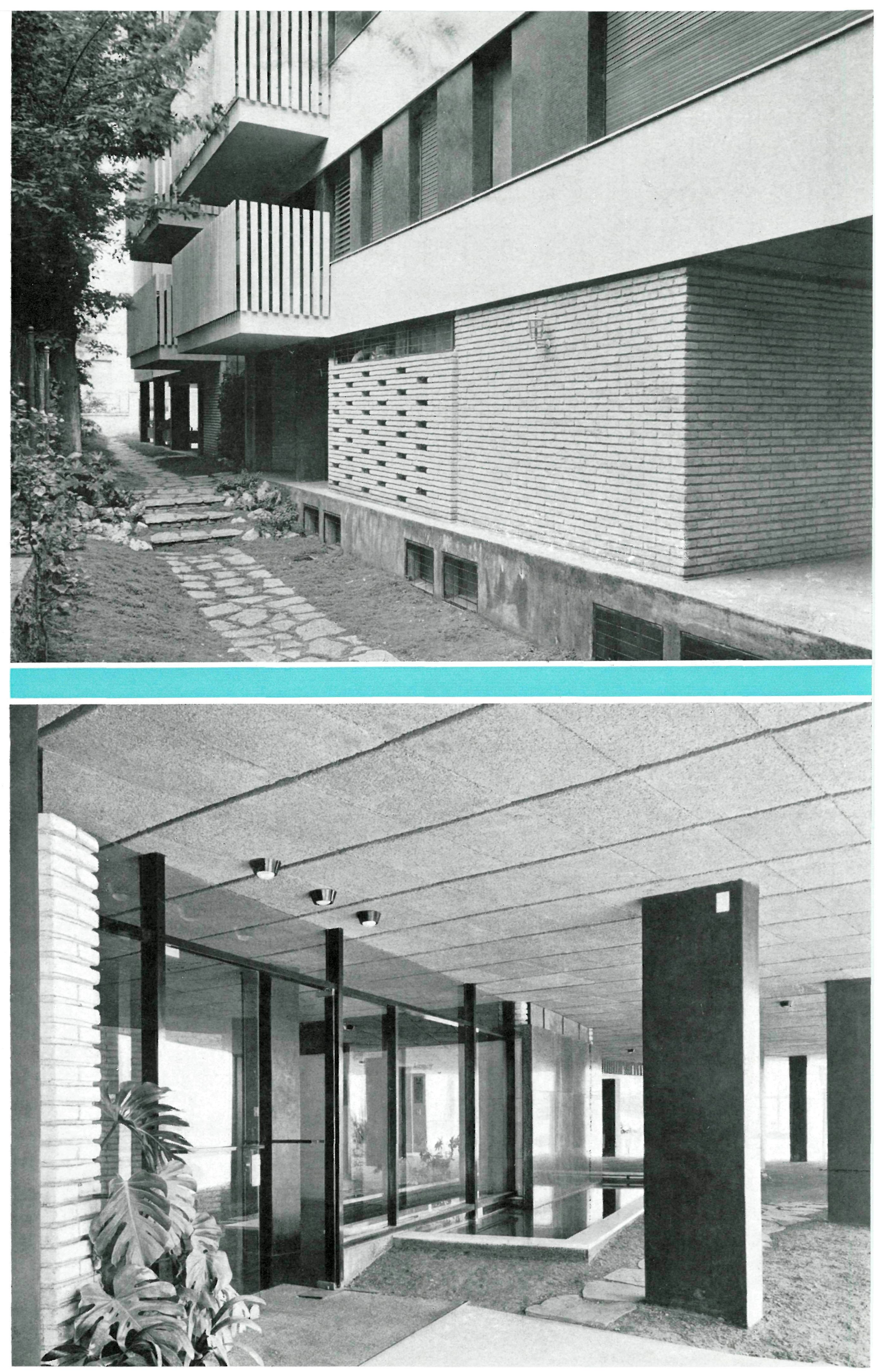


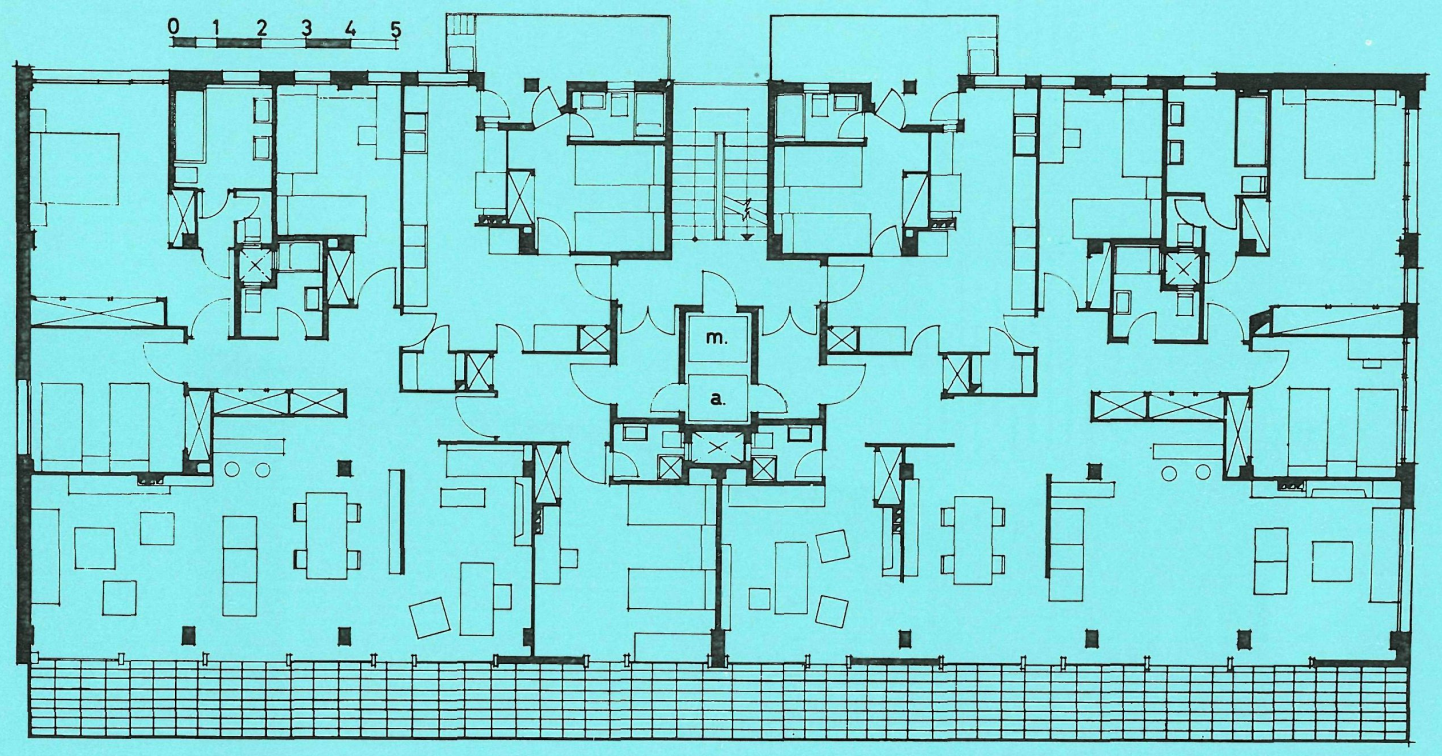

planta

tipo

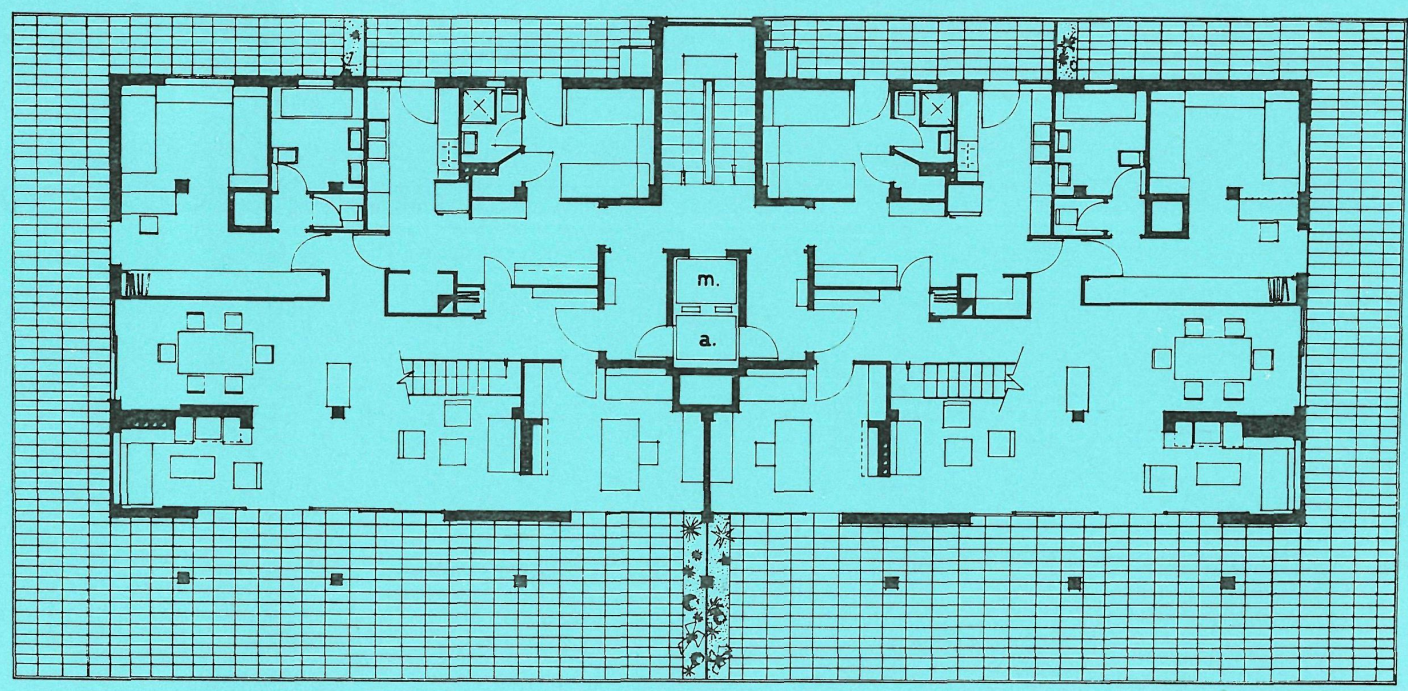

planta de ático

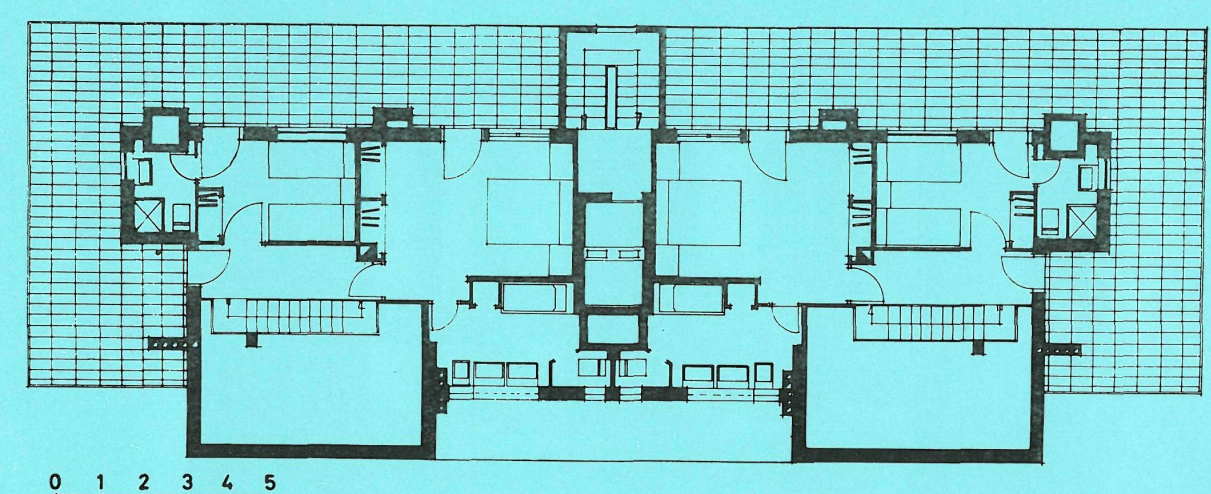

planta
de solbreático 
La superficie construida de cada vivienda es aproximadamente de unos $200 \mathrm{~m}^{2}$; la vivienda tipo, que cubre las necesidades de una familia ya constituida y completa, consta de tres dormitorios dobles, uno de servicio doble y una habitación estudio y posible habitación de invitados, además de las otras zonas de vida en común y servicio; es decir, satisface un programa para el tipo de familia más corriente.

En cuanto a la solución adoptada para la planta tipo: señalaremos que, partiendo de un eje de simetría, está situada la escalera en la parte posterior de dicho eje, que ha sido tratada como escalera de servicio por entender que el acceso a las viviendas se realiza lógicamente a través del ascensor, y que normalmente sólo utilizará la escalera el servicio; por este motivo se le dio una cierta dignidad, pero prescindiendo de toda pretensión. Como puede verse en la planta, el grupo de ascensores está unido con la escalera, formando un centro de comunicaciones verticales.

Quedan entonces claramente definidos los dos circuitos de paso: uno que desde el ascensor, pasando por el vestíbulo del ascensor, penetra en la vivienda por la zona de relación, para llegar a los dormitorios; y el otro, que del montacargas sale al rellano de escaleras y por el oficio de servicio va a parar también a la misma zona de dormitorios. Con ello, queda totalmente eliminada la necesidad de un pasillo de reparto.

Las separaciones entre la zona de relación y el estudio han sido realizadas a base de paneles de tabiques sueltos, acabados en su parte alta con cristal para conseguir una mayor sensación de volumen y continuidad.

En cuanto a la composición de las fachadas, su parte N. presenta un muro ciego de refractario sin abertura, y han sido abiertas en cambio las fachadas orientadas al S.

Respecto a la fachada principal, orientada al SO., fue proyectada en un principio para defenderse del sol de tarde en verano, con unas persianas plegables que se recogían en la parte
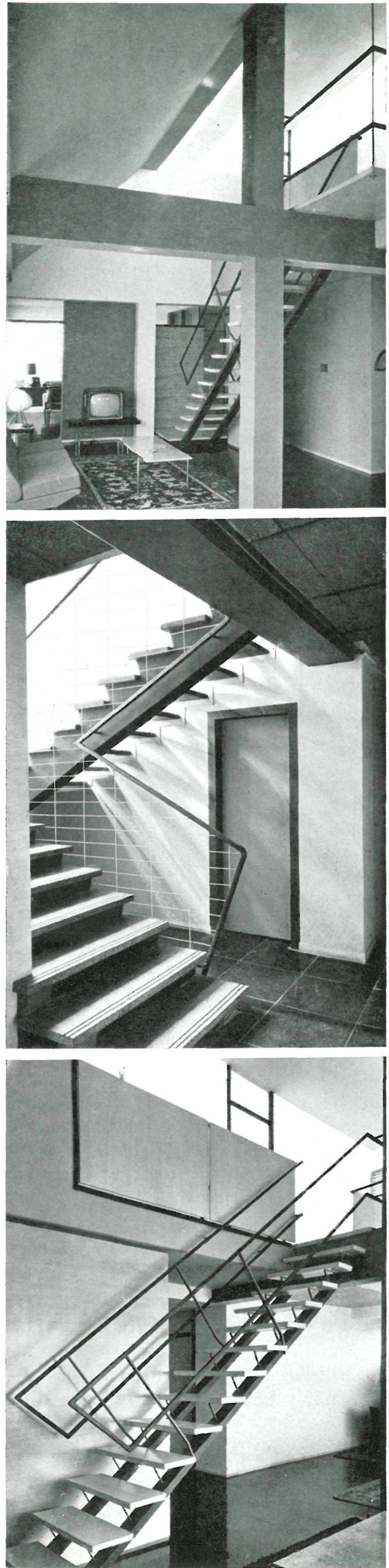


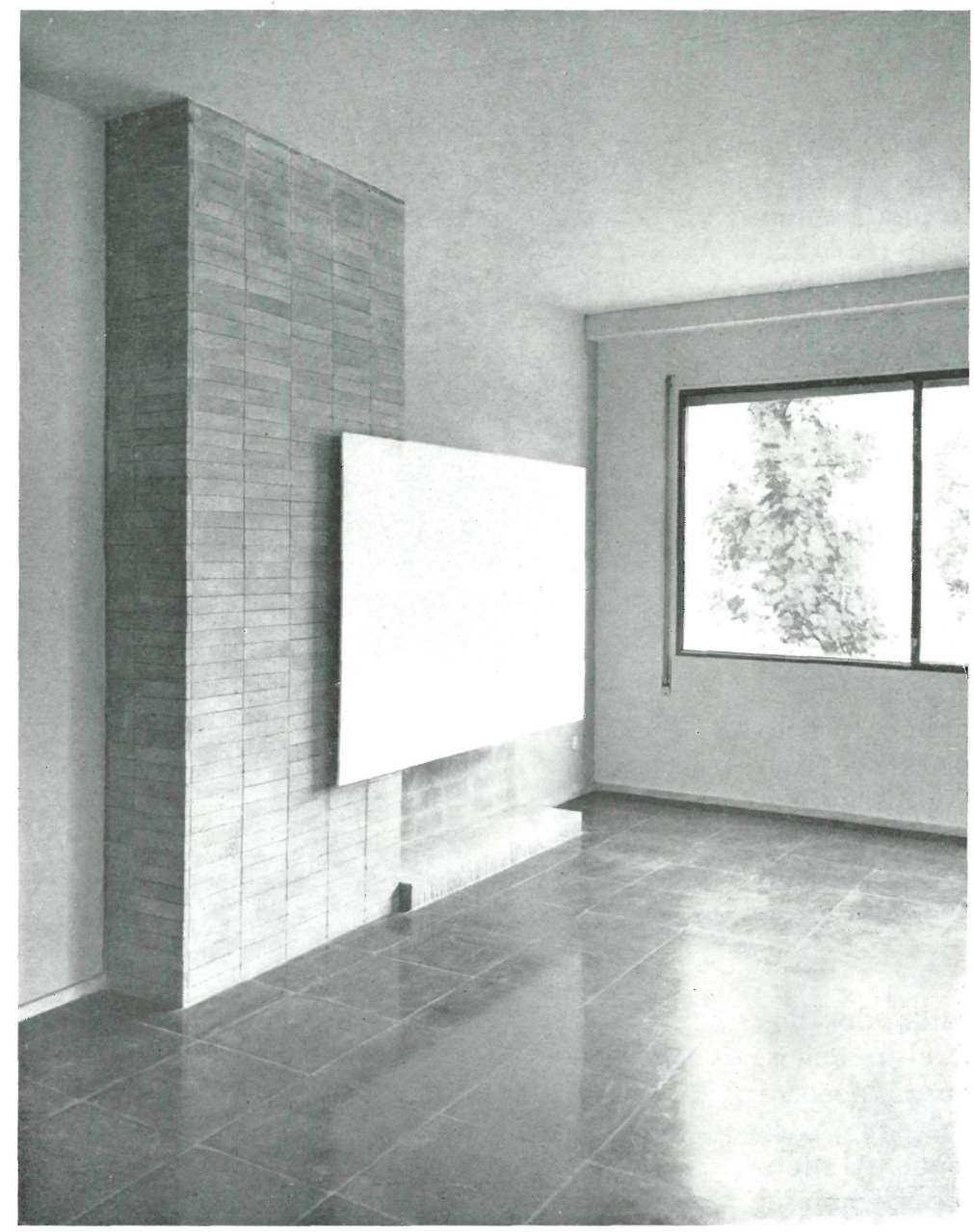

alta y dentro de unas petacas de hierro situadas debajo de las canales de plomo de recogidas de agua y frente a los voladizos. Esta solución tenía además el atractivo de que la terraza quedaba prácticamente enlazada con la vivienda, aumentando la sensación de superficie al cerrar al borde mismo de las terrazas. Pero por razones económicas fueron suprimidas.

Las barandas permiten a las personas estar en la terraza sin ser vistas desde el exterior, pudiendo en cambio ver la calle desde la terraza.

La zona de tendido se halla oculta por unas lamas verticales de uralita.

El ático y sobreático están enlazados formando un «Duplex».

El edificio constituye un ejemplo de sencillez constructiva, de elegantes líneas y distribución racional y cuidadosamente pensada.

El cálculo de la estructura se confió al ingeniero Gunter Oistrach. 


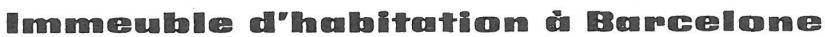

F. Barba Corsini, architecte. J. Cánoves et M. Casanellas, architectes collaborateurs

Cet immeuble comprend, au sous-sol, un garage et, au rez-de-chaussée, une zone de jeux commune à tous les enfants de l'édifice, en plus des vestibules. Le reste de la surface édifiée est destiné aux logements, de $200 \mathrm{~m}^{2}$ environ, dont la distribution fonctionnelle s'adapte aux nécessités des familles moyennes.

Une attention spéciale a été attachée à la composition des façades, qui offrent, parmi d'autres attraits, celui de leur élégante simplicité.

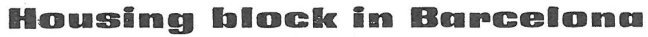

F. Barba Corsini, architect. J. Cánoves and M. Casanellas, collaborating architects

This building has a garage basement, a ground floor with a communal playing zone for all the children of the block, and the entrance vestibule. The rest of the building is occupied with apartments, of about $200 \mathrm{~m}^{2}$ each, which are functionally designed, and are suitable for medium sized average income families.

Special attention has been given to the external facades, which, among other things, are of extremeiy simple design.

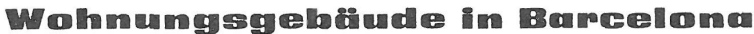

F. Barba Corsini, Architekt. J. Cánoves und M. Casanellas, Mitarbeiter-Architekten

Das Gebäude enthält: im Untergeschosse eine Garage; im Erdgeschosse eine Spielzone neben den Vorhallen für die Kinder von allen Wohnungen; die gebaute Restoberfläche ist zu Wohnungen von $200 \mathrm{~m}^{2}$ bestimmt. Sie sind mit einer geeigneten Verteilung für Mittelstandsfamilien gebaut.

Besonders beachtet man die Komposition der Fassaden, die ihre elegante Einfachheit bietet. 